\title{
ON THE FACTORIZATION METHOD FOR A FAR FIELD INVERSE SCATTERING PROBLEM IN THE TIME DOMAIN*
}

\author{
FIORALBA CAKONI ${ }^{\dagger}$, HOUSSEM HADDAR ${ }^{\ddagger}$, AND ARMIN LECHLEITER $^{\S}$
}

\begin{abstract}
We develop a factorization method to obtain explicit characterization of a (possibly non-convex) Dirichlet scattering object from measurements of time-dependent causal scattered waves in the far field regime. In particular, we prove that far fields of solutions to the wave equation due to particularly modified incident waves, characterize the obstacle by a range criterion involving the square root of the time derivative of the corresponding far field operator. Our analysis makes essential use of a coercivity property of the solution of the Dirichlet initial boundary value problem for the wave equation in the Laplace domain. This forces us to consider this particular modification of the far field operator. The latter in fact, can be chosen arbitrarily close to the true far field operator given in terms of physical measurements.
\end{abstract}

In memory of our dearest colleague, friend and the co-author of this paper, Armin Lechleiter! F.C. and H.H.

Key words. Factorization method, inverse scattering, wave equation

AMS subject classifications. 35J25, 35P25, 35Q60; 35R30

1. Introduction. Reconstructing the shape of an obstacle from measurements of time-dependent scattered waves is an important classical inverse scattering problem with many potential applications such as in non-destructive testing and medical imaging by ultrasound waves. Commonly used inversion methods, such as Kirchhoff or travel time migration (see for instance $[6,8,9]$ and references therein), are usually based on high frequency or weak scattering approximations. More recently, new families of imaging techniques that avoid these approximations by relying on the use of multi-static measurements have been proposed (see for instance [1, 11, 12, 16, 29, 36] and references therein). They are commonly referred to as sampling methods. However, most of these techniques have been developed only in the frequency domain. One of the prominent members of this family is the so-called factorization method $[27,29]$. The main advantage of this method, as opposed to other sampling techniques, is that it yields a mathematically rigorous characterization of the scatterer's shape in terms of the data. Therefore, in addition to suggesting a fast numerical inversion algorithm that is justified for noisy data, it also implies a uniqueness result for the associate inverse problem. Designing a mathematically justified sampling method, in particular a factorization method, in the time domain is still an open problem. Our intention here is to provide a theoretical framework that would help clarifying why this is a hard problem. Indeed here we prove the factorization method for a (small) perturbation of the far field operator that is more conducive to analyzing the problem using the Laplace transform instead of the Fourier transform. In order to ensure a symmetric factorization that is a fundamental requirement for the method, we consider waves associated with "conjugated" wave numbers. The latter transforms in the

* The research of F.C. was supported in part by the AFOSR Grant FA9550-17-1-0147, NSF Grants DMS-1602802 and DMS-1813492.

${ }^{\dagger}$ Department of Mathematics, Rutgers University, Piscataway, NJ 08854, USA (fc292@math.rutgers.edu)

${ }^{\ddagger}$ INRIA Saclay Ile de France / CMAP Ecole Polytechnique, Route de Saclay, 91128 Palaiseau Cedex, France (houssem.haddar@polytechnique.edu).

$\S$ Deceased, Center for Industrial Mathematics, University of Bremen, Bremen, Germany 
time domain as a multiplicative factor involving the imaginary part of the frequency. Hence our result should be seen as step forward toward the goal of developing a time domain mathematically justified linear sampling type method.

What is the benefit of developing the factorization method in the time domain? In fact, linear sampling methods can be formulated at a single frequency, but it is well-known that to achieve reasonable reconstruction they need multistatic data on a large spatial aperture. On the other hand, the resolution of reconstructions heavily depends on the interrogating frequency, and for a discrete set of frequencies (interior eigenvalues) these methods do not work, which becomes an issue because good or bad frequencies depend on the unknown scatterer. Using time-domain data can be a remedy for these issues. In fact preliminary numerical results using the linear sampling method indicate that using time domain data reduces the spacial aperture as well as the number of receivers/transmitters without compromising the quality of the reconstructions $[13,14,15,21,22,24]$. When given time-dependent scattering data, one might also be tempted to take formal Fourier transforms of the data, and then to apply single frequency reconstruction methods at several frequencies. The paper [23] shows that this can lead to numerical difficulties for sampling methods. In addition, superimposing single frequencies images does not respect the causality property of the fields. Thus the natural way to handle time-domain data is to develop reconstruction methods in the framework of time-dependent wave equation. A first attempt to consider sampling methods (more precisely, the linear sampling method) in a somewhat different time-dependent setting was made in [15]. Unfortunately, the method proposed in [15] and subsequently in [22, 24], does not provide a rigorous mathematical characterization of the obstacle, due to an approximation argument used in the mathematical justification of the method (see [12] for the time-harmonic case). The characterization provided in the present paper is designed for the far field full aperture setting as opposed to the near-field (possibly partial aperture) setting considered in [15]. The far field full aperture setting introduces additional mathematical structure that allows to go beyond the results of [15]. Roughly speaking, since incident and scattered fields are only adjoint if one additionally reverses time, in the near field setting or partial aperture one loses symmetry which in turn determines important factorization properties of the measurement operator. We also mention the work in [39] as an attempt to develop a factorization method in the time domain for the Robin problem.

Finally, we would like to mention other works related to inverse problems for waves in the time domain. Apart from the above mentioned sampling methods, other techniques for inverse scattering problems, the namely probe method and the pointsource method $[10,32]$ as well as the enclosure method $[25,26]$ also have been extended to time domain problems. Furthermore, many authors investigated time reversal techniques, partially linked with control theoretic approaches, see, e.g. $[5,7$, $9,17,30,33,34,35]$. It is worth to note that many of these results rely on geometric assumptions for the obstacle, whereas we do only suppose that the scatterer is a Lipschitz domain with connected complement. Of course, the price to pay is that our characterization requires measurements of the causal wave for all (positive) times.

The outline of the paper is as follows. In the next section, we formulate the direct and inverse scattering problem for the wave equation with a Dirchlet obstacle, and define the concept of the far field pattern for causal waves as well as the time domain far field operator. In Section 3, we introduce the time domain retarded Herglotz function, derive the basic factorization of the far field operator and define the analytical 
framework to study this factorization. The middle operator in the factorization is related to the solution operator of the initial boundary value problem, which we study in terms of retarded potentials. Here we recall important results on the properties of these potentials due to Bamberger and Ha Duong in [4], which are obtained by intermediately using the Laplace transform framework. Section 4 is dedicated to deriving our main inversion result, which is stated in Theorem 4.5. In particular, we show that in order to obtain a symmetric factorization, we need to consider the far fields due to modified incident waves. The latter are the inverse Laplace transform of entire solutions to the Helmholtz equation with complex wave with negative imaginary part. The corresponding far fields give rise to a perturbed far field operator, which assumes a symmetric factorization with coercive middle operator leading to the proof of the factorization method. The Appendix contains some abstract known results from the literature that we use in our proofs.

The first two authors F.C. and H.H would like to add the following statement: We dedicate this paper to the memory of Professor Armin Lechleiter, with whom we started working on this project in 2017. Professor Armin Lechleiter prematurely passed away in January 2018 at the age of 35. Collaborating with Armin was a most pleasant, memorable experience and an intellectual challenge. His loss will be strongly felt by all colleagues and friends who had the privilege to know him personally.

2. Problem Setting for Dirichlet Obstacles. We consider a Dirichlet scattering object $D \subset \mathbb{R}^{3}$ that we suppose to be a Lipschitz domain. The obstacle $D$ is allowed to possess several components, however, the exterior $\mathbb{R}^{3} \backslash \bar{D}$ of $D$ is assumed to be connected. Without loss of generality we suppose that $D$ contains the origin. Wave propagation in $\mathbb{R}^{3} \backslash \bar{D}$ is described by the wave equation

$$
\partial_{t}^{2} u-\Delta u=0 \quad \text { in } \mathbb{R}^{3} \backslash \bar{D} \times \mathbb{R},
$$

subject to a Dirichlet boundary condition on $\partial D$, and such that $u$ vanishes for $t \leq T$. Here, $T \in \mathbb{R}$ is a given "initial" time. Given an incident wave $u^{i}(x, t)$ solving the wave equation in $\mathbb{R}^{3} \times \mathbb{R}$, and such that the restriction $\left.u^{i}\right|_{\partial D}$ vanishes for $t \leq T$, the scattered field is defined as $u^{s}:=u-u^{i}$. This wave field solves the direct scattering problem (2.1) together with the boundary condition $u^{s}=-u^{i}$ on $\partial D$ and the causality condition $u^{s}(x, t)=0$ for $t \leq T$.

For causal solutions to the wave equation there exists the notion of an associated far field. Roughly speaking, the far field describes the behavior of the wave far away from the scatterer. Due to the time-dependence of the wave, the far field of a scattered wave depends on a direction $\xi \in \mathbb{S}^{2}:=\left\{\theta \in \mathbb{R}^{3},|\theta|=1\right\}$, and on a time variable $t \in \mathbb{R}$. Analysis of the far field of solutions to the wave equation goes back to Friedlander [18, 19]. For instance, in [18] it is shown that a twice continuously differentiable solution $u^{s}$ to $(2.1)$ satisfies

$$
\lim _{r \rightarrow \infty} r u^{s}(r \xi, r+t)=u^{\infty}(\xi, t) \quad \text { for } \xi \in \mathbb{S}^{2} \text { and } t \in \mathbb{R},
$$

for a function $u^{\infty}: \mathbb{S}^{2} \times \mathbb{R} \rightarrow \mathbb{R}$ called the far field of $u^{s}$.

We shall here formally explain the setting of the inverse scattering problem. We use incident waves in the form of wave fronts,

$$
u^{i}(x, t ; \theta):=\delta(t-\theta \cdot x),
$$

where $\theta \in \mathbb{S}^{2}$ is a given direction. These distributional solutions to the wave equation then formally satisfy the causality condition with $T<-d$. We formally associate a 
far field $u^{\infty}(\xi, t ; \theta)$ to these incident fields. The inverse problem is to reconstruct the geometry $D$ from the knowledge of $u^{\infty}(\xi, t ; \theta)$ on $\mathbb{S}^{2} \times \mathbb{R} \times \mathbb{S}^{2}$.

Central to our method is the far field operator $F$ defined (at least formally) as

$$
(F g)(\xi, t):=\int_{\mathbb{R}} \int_{\mathbb{S}^{2}} u^{\infty}\left(\xi, t-t_{0} ; \theta\right) g\left(\theta, t_{0}\right) \mathrm{d} \theta \mathrm{d} t_{0} \quad \text { for } \xi \in \mathbb{S}^{2} \text { and } t \in \mathbb{R}
$$

and for regular functions $g \in C_{0}^{\infty}\left(\mathbb{S}^{2} \times \mathbb{R}\right)$. Using the linearity of the forward problem, this operator maps densities $g$ to the far field pattern $u^{\infty}$ associated to the incident field

$$
v_{g}(x, t)=\int_{\mathbb{R}} \int_{\mathbb{S}^{2}} \delta\left(t-t_{0}-\theta \cdot x\right) g\left(\theta, t_{0}\right) \mathrm{d} \theta \mathrm{d} t_{0}=\int_{\mathbb{S}^{2}} g(\theta, t-\theta \cdot x) \mathrm{d} \theta .
$$

As indicated in the introduction, we shall prove a characterization of the domain $D$ in terms of a modified far field operator that can be arbitrarily close to the physical far field operator $F$.

3. Retarded Potentials and Solutions to the Wave Equation. Our analysis of direct and inverse time domain scattering problems relies on retarded potentials, and we would like to recall standard results concerning the retarded single-layer potential. These results give a rigorous solution theory for exterior wave propagation problems, which will allow us (in the beginning of Section 3.3) to rigorously define the far field operator on smooth functions with compact support.

Let us recall that $k(x, t)=\delta(t-|x|) / 4 \pi|x|$ is the fundamental solution for the wave equation in three dimensions [38]. Using this fundamental solution we can formally introduce layer potentials. Define the single-layer potential on $\partial D$ by

$$
\begin{aligned}
(\mathrm{SL} \psi)(x, t) & =\int_{\mathbb{R}} \int_{\partial D} k\left(x-y, t-t_{0}\right) \psi\left(y, t_{0}\right) \mathrm{d} s(y) \mathrm{d} t_{0} \\
& =\int_{\partial D} \frac{\psi(y, t-|x-y|)}{4 \pi|x-y|} \mathrm{d} s(y) \quad \text { for } x \in \mathbb{R}^{3} \backslash \partial D \text { and } t \in \mathbb{R} .
\end{aligned}
$$

The corresponding single-layer operator is

$$
(S \psi)(x, t)=\int_{\partial D} \frac{\psi(y, t-|x-y|)}{4 \pi|x-y|} \mathrm{d} s(y) \quad \text { for } x \in \partial D \text { and } t \in \mathbb{R} .
$$

The importance of these potentials is obvious from the fact that for a given incident wave $u^{i}(x, t)$, the scattered wave $u^{s}$ is given by

$$
u^{s}=-\mathrm{SL}\left[S^{-1}\left(\left.u^{i}\right|_{\partial D}\right)\right] \quad \text { in } \mathbb{R}^{3} \backslash \bar{D} \times \mathbb{R} .
$$

We briefly recall main theoretical results for the direct scattering problem, based on Laplace transform techniques [4], [31].

For a Hilbert space $X$ we denote by $\mathcal{D}(\mathbb{R} ; X)=C_{0}^{\infty}(\mathbb{R} ; X)$ smooth and compactly supported $X$-valued functions. Further, $\mathcal{D}^{\prime}(\mathbb{R} ; X)$ are $X$-valued distributions on the real line and the corresponding tempered distributions are $\mathcal{S}^{\prime}(\mathbb{R} ; X)$. We also set

$$
\mathcal{L}^{\prime}(\mathbb{R} ; X):=\left\{f \in \mathcal{D}^{\prime}(\mathbb{R} ; X), e^{-\sigma_{f} t} f(t) \in \mathcal{S}^{\prime}(\mathbb{R} ; X) \text { for some } \sigma_{f} \in \mathbb{R}\right\} .
$$

The Fourier-Laplace transform of $f \in \mathcal{L}^{\prime}(\mathbb{R} ; X)$ is

$$
\mathcal{L}[f](s):=\int_{-\infty}^{\infty} e^{\mathrm{i} s t} f(t) \mathrm{d} t \quad s=\omega+\mathrm{i} \sigma \quad \text { for } \sigma>\sigma_{f},
$$


If $\sigma=0$, then the Laplace transform coincides with the usual Fourier transform. We introduce for each $m \in \mathbb{R}$ the Hilbert spaces

$$
H_{\sigma}^{m}(\mathbb{R} ; X):=\left\{f \in \mathcal{L}^{\prime}(\mathbb{R} ; X), \int_{-\infty+\mathrm{i} \sigma}^{\infty+\mathrm{i} \sigma}|s|^{2 m}\|\mathcal{L}[f](s)\|_{X}^{2} \mathrm{~d} s<\infty\right\}
$$

for $m \in \mathbb{R}$ and $\sigma \in \mathbb{R}$, endowed with the norm

$$
\|f\|_{H_{\sigma}^{m}(\mathbb{R}, X)}=\left(\int_{-\infty+\mathrm{i} \sigma}^{\infty+\mathrm{i} \sigma}|s|^{2 m}\|\mathcal{L}[f](s)\|_{X}^{2} d s\right)^{1 / 2}
$$

and obvious inner product.

Denote by $X^{*}$ the dual space of $X$ with a duality pairing denoted by $\langle,\rangle_{X^{*}, X}$. We then clearly see that $H_{\sigma}^{-m}\left(\mathbb{R}, X^{*}\right)$ can be identified with the dual space of $H_{\sigma}^{m}(\mathbb{R}, X)$ with respect to the duality pairing

$$
\langle f, g\rangle=\int_{-\infty+\mathrm{i} \sigma}^{\infty+\mathrm{i} \sigma}\langle\mathcal{L}[g](s), \mathcal{L}[f](s)\rangle_{X^{*}, X} d s=\int_{-\infty}^{\infty} e^{-2 \sigma t}\langle g(t), f(t)\rangle_{X^{*}, X} d t
$$

For $T \in \mathbb{R}$ we define

$$
H_{\sigma}^{m}\left(\mathbb{R}_{>T} ; X\right)=\left\{f \in H_{\sigma}^{m}(\mathbb{R} ; X) \text {, such that } f(t)=0 \text { for } t<T\right\}
$$

which is a closed subspace of $H_{\sigma}^{m}(\mathbb{R} ; X)$ and

$$
\tilde{H}_{\sigma}^{m}\left(\mathbb{R}_{>T} ; X\right)=\left\{\left.f\right|_{t \geq T}, f \in H_{\sigma}^{m}(\mathbb{R} ; X)\right\}
$$

endowed with the quotient norm (see e.g. Rudin1991). For $T \in \mathbb{R}, m \geq 0$ and $\sigma>0$, we have the following inclusions

$$
H_{\sigma}^{m}\left(\mathbb{R}_{>T} ; X\right) \subset L_{\sigma}^{2}\left(\mathbb{R}_{>T} ; X\right) \subset H_{\sigma}^{-m}\left(\mathbb{R}_{>T} ; X\right) \subset \tilde{H}_{\sigma}^{-m}\left(\mathbb{R}_{>T} ; X\right) .
$$

Moreover, $\tilde{H}_{\sigma}^{-m}\left(\mathbb{R}_{>T} ; X^{*}\right)$ can be identified with the dual space of $H_{\sigma}^{m}\left(\mathbb{R}_{>T} ; X\right)$ with respect to the duality pairing defined in (3.4). Now we assume that $H$ is a Hilbert pivot space in the duality $X^{*}, X$, i.e. $X \subset H \subset X^{*}$ with dense inclusions and that the duality pairing coincide with the inner product associated with $H$. Then we also have that

$$
H_{\sigma}^{m}\left(\mathbb{R}_{>T} ; X\right) \subset L_{\sigma}^{2}\left(\mathbb{R}_{>T} ; H\right) \subset \tilde{H}_{\sigma}^{-m}\left(\mathbb{R}_{>T} ; X^{*}\right)
$$

and the three spaces form a Gelfand triple with a pivot space $L_{\sigma}^{2}\left(\mathbb{R}_{>T} ; H\right)$.

The following theorem is proven in [4] (see also [38]).

Theorem 3.1 (Bamberger and Ha Duong, 1986). Let $m \in \mathbb{R}, \sigma>0$, and $T \in \mathbb{R}$.

(i) The single-layer operator $S$ is invertible and the inverse

$$
S^{-1}: H_{\sigma}^{m}\left(\mathbb{R}_{>T} ; H^{1 / 2}(\partial D)\right) \rightarrow H_{\sigma}^{m-2}\left(\mathbb{R}_{>T} ; H^{-1 / 2}(\partial D)\right)
$$

is bounded. The single-layer potential

$$
\mathrm{SL}: H_{\sigma}^{m}\left(\mathbb{R}_{>T} ; H^{-1 / 2}(\partial D)\right) \rightarrow H_{\sigma}^{m-1}\left(\mathbb{R}_{>T} ; H^{1}\left(\mathbb{R}^{3}\right)\right)
$$

is bounded.

(ii) For boundary data $h \in H_{\sigma}^{m}\left(\mathbb{R}_{>T} ; H^{1 / 2}(\partial D)\right)$ there is a unique solution $u=$ $\mathrm{SL}\left(S^{-1} g\right)$ in $H_{\sigma}^{m-3 / 2}\left(\mathbb{R}_{>T} ; H^{1}\left(\mathbb{R}^{3} \backslash \bar{D}\right)\right)$ of the boundary value problem $\partial_{t}^{2} u-\Delta u=0$ in $\left(\mathbb{R}^{3} \backslash \bar{D}\right) \times \mathbb{R}, u=h$ on $\partial D \times \mathbb{R}$, and $u=0$ for $t \leq T$. 
3.1. Retarded Herglotz Waves. The first step towards a rigorous definition of the far field operator and its mapping properties, is to study the properties of the incident retarded Herglotz wave functions $v_{g}$ defined in (2.3). We remark that $v_{g}$ is a regular solution to the wave equation in $\mathbb{R}^{3} \times \mathbb{R}$, at least for smooth and compactly supported $g$. Recall that $d=\sup _{x \in D}|x|$.

Lemma 3.2. Let $m \in \mathbb{R}, l \in \mathbb{N}_{0}$ and $\sigma>0$. Then the mapping $\left.g \mapsto v_{g}\right|_{D}$ is bounded from $H_{\sigma}^{m+l}\left(\mathbb{R} ; L^{2}\left(\mathbb{S}^{2}\right)\right)$ into $H_{\sigma}^{m}\left(\mathbb{R} ; H^{l}(D)\right)$. If $T \in \mathbb{R}$ and $g(\cdot, t)$ vanishes for $t<T$, then $v_{g}(\cdot, t)$ vanishes in $D$ for $t<T-d$.

Proof. For smooth and compactly supported $g \in C_{0}^{\infty}\left(\mathbb{R} ; C^{\infty}\left(\mathbb{S}^{2}\right)\right)$, the application of the Laplace transform and Fubini's theorem yield that

$$
\begin{aligned}
\mathcal{L}\left[v_{g}\right](k) & =\int_{\mathbb{R}} \exp (\mathrm{i} k t) \int_{\mathbb{S}^{2}} g(\theta, t-\theta \cdot x) \mathrm{d} \theta \mathrm{d} t \\
& =\int_{\mathbb{S}^{2}} \exp (\mathrm{i} k \theta \cdot x) \int_{\mathbb{R}} \exp (\mathrm{i} k r) g(\theta, r) \mathrm{d} r \mathrm{~d} \theta=\int_{\mathbb{S}^{2}} \exp (\mathrm{i} k \theta \cdot x) \mathcal{L}[g](\theta, k) \mathrm{d} \theta
\end{aligned}
$$

for $k:=\omega+\mathrm{i} \sigma, \omega \in \mathbb{R}$ and fixed $\sigma>0$. The product rule shows that

$$
\begin{aligned}
\partial_{k}\left(k^{\beta} \mathcal{L}\left[v_{g}\right](k)\right)=\beta k^{\beta-1} \int_{\mathbb{S}^{2}} \exp (\mathrm{i} k \theta \cdot x) \mathcal{L}[g](\theta, k) \mathrm{d} \theta \\
\quad+k^{\beta} \int_{\mathbb{S}^{2}}(\mathrm{i} \theta \cdot x) \exp (\mathrm{i} k \theta \cdot x) \mathcal{L}[g](\theta, k) \mathrm{d} \theta+k^{\beta} \int_{\mathbb{S}^{2}} \exp (\mathrm{i} k \theta \cdot x) \partial_{k} \mathcal{L}[g](\theta, k) \mathrm{d} \theta
\end{aligned}
$$

for $\beta \in \mathbb{N}$. Since $|\exp (\mathrm{i} k \theta \cdot x)| \leq C(\sigma)$ for all $x \in D, \theta \in \mathbb{S}$ and $\omega \in \mathbb{R}$, we conclude that

$\left\|\partial_{k} k^{\beta} \mathcal{L}\left[v_{g}\right](k)\right\|_{H^{l}(D)}^{2} \leq C(\sigma, D) \sum_{j=0}^{l} 2^{j}\left(1+|k|^{2}\right)^{\beta+j}\left(\|\mathcal{L}[g](\cdot, k)\|_{L^{2}\left(\mathbb{S}^{2}\right)}^{2}+\left\|\partial_{k} \mathcal{L}[g](\cdot, k)\right\|_{L^{2}\left(\mathbb{S}^{2}\right)}^{2}\right)$

Due to the definition of $H_{\sigma}^{m}\left(\mathbb{R} ; H^{l}(D)\right)$ and the transformation rules, we estimate that

$$
\left\|v_{g}\right\|_{H_{\sigma}^{m}\left(\mathbb{R} ; H^{l}(D)\right)}^{2} \leq C\|g\|_{H_{\sigma}^{m+l}\left(\mathbb{R} ; L^{2}\left(\mathbb{S}^{2}\right)\right)}^{2}
$$

for smooth $g$ with compact support. This bound extends by density from $C_{0}^{\infty}\left(\mathbb{R} ; C^{\infty}\left(\mathbb{S}^{2}\right)\right)$ to $H_{\sigma}^{m+l}\left(\mathbb{R} ; L^{2}\left(\mathbb{S}^{2}\right)\right)$.

Now, assume that the density $g(\cdot, t)$ vanishes for $t<T$. For $x \in D$ and $t<T-d$ we have $t-\theta \cdot x<T$ for all $\theta \in \mathbb{S}^{2}$ and hence $g(\cdot, t-\theta \cdot x)$ vanishes on $\mathbb{S}^{2}$. Thus, the right-hand side of (2.3) implies that $v_{g}(x, t)$ vanishes for $x \in D$ and $t<T-d$.

Combining the above lemma with the trace theorem from $H^{1}(D)$ into $H^{1 / 2}(\partial D)$ shows that $\left.g \mapsto v_{g}\right|_{\partial D}$ is also bounded from $H_{\sigma}^{m+1}\left(\mathbb{R} ; L^{2}\left(\mathbb{S}^{2}\right)\right)$ into $H_{\sigma}^{m}\left(\mathbb{R} ; H^{1 / 2}(\partial D)\right)$. In what follows, this mapping is called the Herglotz operator and is denoted by

$$
\mathcal{H} g:=\left.v_{g}\right|_{\partial D \times \mathbb{R}} .
$$

REMARK 3.3. If one formally takes a Laplace transform of the retarded Herglotz wave $v_{g}$ from (2.3), then one finds at each $k=\omega+\mathrm{i} \sigma$ a Herglotz wave functions in the Laplace domain with density $\mathcal{L}[g](\cdot, k)$ and complex wave number $k:=\omega+\mathrm{i} \sigma$ (see, e.g., [16] in the case of real wave number). 
3.2. Far Fields Associated with Single-Layer Potentials. To properly define the far field operator we need to extend definition (2.2) of far fields associated to smooth solutions to the wave equation to single-layer potentials with densities that are not regular in time. To this end, let us consider (causal) solutions to the wave equation in form of a single-layer potential,

$$
v(x, t)=(\operatorname{SL} \psi)(x, t)=\int_{\partial D} \frac{\psi(y, t-|x-y|)}{4 \pi|x-y|} \mathrm{d} s(y) \quad \text { for } x \in \mathbb{R}^{3} \backslash \bar{D} \text { and } t \in \mathbb{R} .
$$

Lemma 3.4. Let $\psi \in C_{0}^{\infty}\left(\mathbb{R} ; C^{\infty}(\partial D)\right)$ and define $v$ by (3.6). Then

$$
\lim _{r \rightarrow \infty} r v(r \xi, r+t)=\frac{1}{4 \pi} \int_{\partial D} \psi(y, t+\xi \cdot y) \mathrm{d} s(y) \quad \text { for } \xi \in \mathbb{S}^{2} \text { and } t \in \mathbb{R} .
$$

Proof. For smooth and compactly supported $\psi$ it holds that

$$
\lim _{r \rightarrow \infty} r v(r \xi, r+t)=\lim _{r \rightarrow \infty} \int_{\partial D} \frac{1}{4 \pi} \frac{r}{|r \xi-y|} \psi(y, r+t-|r \xi-y|) \mathrm{d} s(y) .
$$

However, for $r>2 \sup _{x \in D}|x|$,

$$
\begin{aligned}
& \left|1-\frac{r}{|r \xi-y|}\right|=\left|\frac{|\xi-y / r|-1}{|\xi-y / r|}\right|=\left|\frac{|\xi-y / r|^{2}-1}{|\xi-y / r|(|\xi-y / r|+1)}\right| \\
& \leq\left|\frac{|\xi-y / r|^{2}-1}{|\xi-y / r|}\right| \leq 2|| \xi-y /\left.r\right|^{2}-1|\leq 4| \xi \cdot y|/ r+2| y /\left.r\right|^{2} \leq C(|y|) / r .
\end{aligned}
$$

Moreover,

$$
r-|r \xi-y|=\frac{2 r \xi \cdot y-|y|^{2}}{r+|r \xi-y|}=\xi \cdot y+\xi \cdot y\left(\frac{1-|\xi-y / r|}{1+|\xi-y / r|}\right)-\frac{|y|^{2}}{r+|r \xi-y|} .
$$

Hence, $|r-| r \xi-y|-\xi \cdot y| \leq C(|y|) / r$, which implies (3.7).

Thus we can consider the far field mapping

$$
R: \psi \mapsto \frac{1}{4 \pi} \int_{\partial D} \psi(y, t+\xi \cdot y) \mathrm{d} s(y)
$$

Motivated by (2.2), we call $u^{\infty}=R \psi$ the far field pattern of a retarded single-layer potential $u=\mathrm{SL} \psi$. Note that the formal application of the Fourier transform to $R \psi$ yields simply the time-harmonic far field pattern of a time-harmonic single-layer potential.

Lemma 3.5. For $m \in \mathbb{R}, \sigma>0$ the mapping $R$ defined by (3.8) extends to a bounded operator from $H_{\sigma}^{m}\left(\mathbb{R} ; H^{-1 / 2}(\partial D)\right)$ into $H_{\sigma}^{m-1}\left(\mathbb{R} ; L^{2}\left(\mathbb{S}^{2}\right)\right)$. Furthermore, if $\psi \in H_{\sigma}^{-m}\left(\mathbb{R} ; H^{-1 / 2}(\partial D)\right)$ vanishes for $t<T$, then $R \psi$ vanishes for $t<T-d$.

Proof. For smooth and compactly supported $\psi \in C_{0}^{\infty}\left(\mathbb{R} ; C^{\infty}\left(\mathbb{S}^{2}\right)\right)$, the application of the Laplace transform and Fubini's theorem yield that

$$
\begin{aligned}
\mathcal{L}[R \psi](k) & =\int_{\mathbb{R}} \exp (\mathrm{i} k t) \int_{\partial D} \psi(x, t+\theta \cdot x) \mathrm{d} s_{x} \mathrm{~d} t \\
& =\int_{\partial D} \exp (-\mathrm{i} k \theta \cdot x) \int_{\mathbb{R}} \exp (\mathrm{i} k r) \psi(x, r) \mathrm{d} r \mathrm{~d} s_{x} \\
& =\int_{\partial D} \exp (-\mathrm{i} k \theta \cdot x) \mathcal{L}[\psi](x, k) \mathrm{d} s_{x}
\end{aligned}
$$


for $k:=\omega+\mathrm{i} \sigma, \omega \in \mathbb{R}$ and $\sigma>0$. Then the estimate

$$
\left|\int_{\partial D} \exp (-\mathrm{i} k \theta \cdot x) \mathcal{L}[\psi](x, k) \mathrm{d} s_{x}\right| \leq C(\sigma)|k|\|\mathcal{L}[\psi](\cdot, k)\|_{H^{-1 / 2}(\partial D)}
$$

implies the results. $\square$

The operator $R$ (multiplied by $4 \pi$ ) is the formal adjoint of $H$ for the inner product of $L^{2}\left(\mathbb{R} ; L^{2}\left(\mathbb{S}^{2}\right)\right)$, since

$$
\begin{array}{rl}
\int_{\partial D} \int_{\mathbb{R}} & \mathcal{H} g \psi \mathrm{d} t \mathrm{~d} s(y)=\int_{\mathbb{S}^{2}} \int_{\mathbb{R}} \int_{\partial D} g(\theta, t-\theta \cdot y) \psi(y, t) \mathrm{d} s(y) \mathrm{d} t \mathrm{~d} \theta \\
=\int_{\mathbb{S}^{2}} \int_{\mathbb{R}} g(\theta, r) \int_{\partial D} \psi(y, r+\theta \cdot y) \mathrm{d} s(y) \mathrm{d} r \mathrm{~d} \theta=4 \pi \int_{\mathbb{S}^{2}} \int_{\mathbb{R}} g R \psi \mathrm{d} t \mathrm{~d} \theta
\end{array}
$$

for smooth functions $\psi$ and $g$ with compact support in time.

3.3. Factorization and Mapping Properties of the Far Field Operator. Consider a smooth density $g \in C_{0}^{\infty}\left(\mathbb{R} ; C^{\infty}\left(\mathbb{S}^{2}\right)\right)$ and let $v_{g}$ be the associated Herglotz wave. The scattered field corresponding to the incident field $v_{g}$, known to exist by Theorem 3.1, is $u^{s}=-\mathrm{SL}(\psi)$ where $\psi:=S^{-1}\left(\left.v_{g}\right|_{\partial D}\right)$. Following Lemma 3.4, we define the far field $u^{\infty}$ associated with $u^{s}$ as $u^{\infty}=-R \psi$. The far field operator $F$ is then defined to map $g$ to $u^{\infty}$, that is, $F: g \mapsto u^{\infty}$. It is immediately clear that the far field operator can be factorized as

$$
F g:=-R S^{-1} \mathcal{H} g \stackrel{(3.10)}{=}-\frac{1}{4 \pi} \mathcal{H}^{*} S^{-1} \mathcal{H} g
$$

at least for smooth densities $g \in C_{0}^{\infty}\left(\mathbb{R} ; C^{\infty}\left(\mathbb{S}^{2}\right)\right)$. We set

$$
G:=R S^{-1}
$$

which in fact is the operator that maps $h \mapsto u_{\infty}$ where $u_{\infty}$ is the far field of the unique causal solution to the boundary value problem $\partial_{t}^{2} u-\Delta u=0$ in $\left(\mathbb{R}^{3} \backslash \bar{D}\right) \times \mathbb{R}$, $u=h$ on $\partial D \times \mathbb{R}$, and $u=0$ for $t \leq T$. Thus we can write

$$
F g=-G \mathcal{H} g .
$$

Proposition 3.6. The far field operator $F$ is well-defined and bounded from $H_{\sigma}^{m+2}\left(\mathbb{R} ; L^{2}\left(\mathbb{S}^{2}\right)\right)$ into $H_{\sigma}^{m-2}\left(\mathbb{R} ; L^{2}\left(\mathbb{S}^{2}\right)\right)$, for $m \in \mathbb{R}, \sigma>0$. In addition, let $\tau \in \mathbb{R}$. Then the truncated far field operator $F^{\tau}:\left.g \mapsto F g\right|_{t \geq \tau}$ defines a bounded map from $H_{\sigma}^{m+2}\left(\mathbb{R}_{>\tau} ; L^{2}\left(\mathbb{S}^{2}\right)\right)$ into $\tilde{H}_{\sigma}^{m-2}\left(\mathbb{R}_{>\tau} ; L^{2}\left(\mathbb{S}^{2}\right)\right)$.

Proof. Thanks to Lemma 3.2, Theorem 3.1, and Lemma 3.5, we know that $\mathcal{H}$ is bounded from $H_{\sigma}^{m}\left(\mathbb{R} ; L^{2}\left(\mathbb{S}^{2}\right)\right)$ into $H_{\sigma}^{m-1}\left(\mathbb{R} ; H^{1 / 2}(\partial D)\right)$, that $S^{-1}$ is bounded from $H_{\sigma}^{m}\left(\mathbb{R} ; H^{1 / 2}(\partial D)\right)$ into $H_{\sigma}^{m-2}\left(\mathbb{R} ; H^{-1 / 2}(\partial D)\right)$, and that $R$ is bounded from $H_{\sigma}^{m}\left(\mathbb{R} ; H^{-1 / 2}(\partial D)\right)$ into $H_{\sigma}^{m-1}\left(\mathbb{R} ; L^{2}\left(\mathbb{S}^{2}\right)\right)$, respectively. Now, the mapping properties of $F^{\tau}$ are immediate consequence of the definitions of $H_{\sigma}^{m+2}\left(\mathbb{R}_{>\tau} ; L^{2}\left(\mathbb{S}^{2}\right)\right)$ and $\tilde{H}_{\sigma}^{m-2}\left(\mathbb{R}_{>\tau} ; L^{2}\left(\mathbb{S}^{2}\right)\right)$.

As part of the above proof, we also have the following mapping properties concerning the solution-to-far field operator $G$.

Proposition 3.7. The solution-to-far field operator $G$ is well-defined and bounded from $H_{\sigma}^{m}\left(\mathbb{R} ; H^{1 / 2}(\partial D)\right)$ into $H_{\sigma}^{m-3}\left(\mathbb{R} ; L^{2}\left(\mathbb{S}^{2}\right)\right)$, for $m \in \mathbb{R}, \sigma>0$. 
4. The Perturbed Far Field Operator. For reasons that will become clear later in our analysis, we need to consider a perturbed form of the far field operator. To this end, we consider the modified Herglotz operator given by

$$
\mathcal{H}_{\sigma}:\left.g \mapsto v_{g}^{\sigma}\right|_{\partial D \times \mathbb{R}}
$$

where

$$
v_{g}^{\sigma}(x, t):=\int_{\mathbb{S}^{2}} g(\theta, t-\theta \cdot x) e^{2 \sigma(\theta \cdot x)} d \theta .
$$

Note that $v_{g}^{\sigma}$ is the time convolution of $\delta(t-\theta \cdot x) e^{2 \sigma(\theta \cdot x)}$ with density $g(\theta, t)$. Following the proof of Lemma 3.2 for fixed $\sigma \in \mathbb{R}$, we have that $\mathcal{H}_{\sigma}: H_{\sigma}^{m}\left(\mathbb{R} ; L^{2}\left(\mathbb{S}^{2}\right)\right) \rightarrow$ $H_{\sigma}^{m-1}\left(\mathbb{R} ; H^{1 / 2}(\partial D)\right)$. In terms of the operator $\mathcal{H}_{\sigma}$ and the solution operator $G$ given by $(3.12)$, we now define the perturbed far field operator $F_{\sigma}: H_{\sigma}^{m+2}\left(\mathbb{R} ; L^{2}\left(\mathbb{S}^{2}\right)\right) \rightarrow$ $H_{\sigma}^{m-2}\left(\mathbb{R} ; L^{2}\left(\mathbb{S}^{2}\right)\right)$ by

$$
F_{\sigma} g=-G \mathcal{H}_{\sigma} g
$$

If we let $u_{\sigma}^{\infty}(\xi, t ; \theta)$ be the far field of the unique causal solution to the boundary value problem $\partial_{t}^{2} u-\Delta u=0$ in $\left(\mathbb{R}^{3} \backslash \bar{D}\right) \times \mathbb{R}, u=-\left.u_{\sigma}^{i}\right|_{\partial D \times \mathbb{R}}$ on $\partial D \times \mathbb{R}$, and $u=0$ for $t \leq T$ where $u_{\sigma}^{i}(x, t ; \theta):=\delta(t-\theta \cdot x) e^{2 \sigma(\theta \cdot x)}, \theta \in \mathbb{S}^{2}$, then for smooth densities $g$ with compact support, the far field operator can formally be written as

$$
\left(F_{\sigma} g\right)(\xi, t):=\int_{\mathbb{R}} \int_{\mathbb{S}^{2}} u_{\sigma}^{\infty}\left(\xi, t-t_{0} ; \theta\right) g\left(\theta, t_{0}\right) d \theta d t_{0} .
$$

We remark that the Laplace transform of this "incident field" is

$$
\mathcal{L}\left[u_{\sigma}^{i}(x, t ; \theta)\right](\theta, k)=e^{(\mathrm{i} k+\sigma)(\theta \cdot x)}=e^{\mathrm{i}(\omega-\mathrm{i} \sigma)(\theta \cdot x)},
$$

where $k=\omega+\mathrm{i} \sigma, \omega \in \mathbb{R}, \sigma>0$, and $\theta \in \mathbb{S}^{2}$. Hence $\mathcal{L}\left[u_{\sigma}^{i}(x, t ; \theta)\right](\theta, k)$ in an entire solution of the Helmholtz equation $\Delta v+(\omega-\mathrm{i} \sigma)^{2} v=0$. On the other hand the Laplace transform of the corresponding scattered field $\mathcal{L}\left[u_{\sigma}^{s}\right](\cdot, k ; \theta)$ it is a radiating solution to the Helmholtz equation $\Delta v+(\omega+\mathrm{i} \sigma)^{2} v=0$ with the far field pattern given by $\mathcal{L}\left[u_{\sigma}^{\infty}\right](\xi, k ; \theta)$ for $\xi \in \mathbb{S}^{2}$. We refer the reader to [40] for the concept of the far field pattern and a proof of the Rellich's lemma for the Helmholtz equation with complex wave number $k=\omega+\mathrm{i} \sigma$ for $\sigma>0$. The Laplace transform of the perturbed far field operator then reads

$$
\mathcal{L}\left[F_{\sigma} g\right](\xi, k)=\int_{\mathbb{S}^{2}} \mathcal{L}[g](\theta, k) \mathcal{L}\left[u_{\sigma}^{\infty}\right](\xi, k ; \theta) d \theta .
$$

The operator $F_{\sigma}$ will play the role of the data operator in our analysis. As $\sigma \rightarrow 0$, we have that $F_{\sigma} g$ approaches $F g$ for smooth compactly supported $g$ due to the fact that $u_{\sigma}^{\infty}$ approaches $u^{\infty}$. Indeed this convergence can be shown to hold in the operator norm, but to carry out a rigorous analysis, one must introduce time dependent Sobolev spaces independent of $\sigma$ in terms of the Fourier Transform.

TheOREM 4.1. Let $\sigma>0$ and let $\widetilde{F}_{\sigma}:=-4 \pi\left(\partial_{t} F_{\sigma}-2 \sigma F_{\sigma}\right)$. Then $\widetilde{F}_{\sigma}$ : $H_{\sigma}^{5 / 2}\left(\mathbb{R} ; L^{2}\left(\mathbb{S}^{2}\right)\right) \rightarrow H_{\sigma}^{-5 / 2}\left(\mathbb{R} ; L^{2}\left(\mathbb{S}^{2}\right)\right)$ and the following factorization hold

$$
\widetilde{F}_{\sigma}=\mathcal{H}_{\sigma}^{*}\left(\partial_{t}\left(S^{-1}\right)-2 \sigma S^{-1}\right) \mathcal{H}_{\sigma}
$$


where $\mathcal{H}_{\sigma}^{*}: H_{\sigma}^{-3 / 2}\left(\mathbb{R} ; H^{-1 / 2}(\partial D)\right) \rightarrow H_{\sigma}^{-5 / 2}\left(\mathbb{R} ; L^{2}\left(\mathbb{S}^{2}\right)\right)$ is the dual of $\mathcal{H}_{\sigma}: H_{\sigma}^{5 / 2}\left(\mathbb{R} ; L^{2}\left(\mathbb{S}^{2}\right)\right) \rightarrow$ $H_{\sigma}^{3 / 2}\left(\mathbb{R} ; H^{1 / 2}(\partial D)\right)$ in the $L_{\sigma}^{2}$-duality product defined in (3.4) and is given by

$$
\mathcal{H}_{\sigma}^{*} \psi(\xi, t)=4 \pi R \psi(\xi, t)=\int_{\partial D} \psi(y, t+\xi \cdot y) \mathrm{d} s(y) .
$$

Proof. First we note that, the mapping properties of the indicated operators are obtained from the above with the choice of $m=5 / 2$. From the definition of the operator $R$ and the factorization (3.12) it is clear that $\partial_{t} G=R \partial_{t}\left(S^{-1}\right)$. The factorization of $\widetilde{F}_{\sigma}$ is then a direct consequence of the definition (4.1) and (3.12). To complete the proof we only need to verify (4.3). To this end, consider $g \in C_{0}^{\infty}\left(\mathbb{R}, L^{2}\left(\mathbb{S}^{2}\right)\right)$ and $\psi \in C_{0}^{\infty}\left(\mathbb{R}, H^{-1 / 2}(\partial D)\right)$.

$$
\begin{gathered}
\left(\mathcal{H}_{\sigma} g, \psi\right)_{L_{\sigma}^{2}\left(\mathbb{R}, L^{2}(\partial D)\right)}=\int_{\partial D} \int_{\mathbb{R}} e^{-2 \sigma t}\left(\mathcal{H}_{\sigma} g\right) \psi \mathrm{d} t \mathrm{~d} s(y) \\
=\int_{\mathbb{S}^{2}} \int_{\mathbb{R}} \int_{\partial D} g(\theta, t-\theta \cdot y) e^{-2 \sigma(t-\theta \cdot y)} \psi(y, t) \mathrm{d} s(y) \mathrm{d} t \mathrm{~d} \theta \\
=\int_{\mathbb{S}^{2}} \int_{\mathbb{R}} g(\theta, r) e^{-2 \sigma r} \int_{\partial D} \psi(y, r+\theta \cdot y) \mathrm{d} s(y) \mathrm{d} r \mathrm{~d} \theta \\
=4 \pi \int_{\mathbb{R}} \int_{\mathbb{S}^{2}} e^{-2 \sigma t} g R \psi \mathrm{d} \theta \mathrm{d} t=\left(g, \mathcal{H}_{\sigma}^{*} \psi\right)_{L_{\sigma}^{2}\left(\mathbb{R}, L^{2}\left(\mathbb{S}^{2}\right)\right)} .
\end{gathered}
$$

Our range test in the following involves the dual the operator $\tilde{F}_{\sigma}^{*}: H_{\sigma}^{5 / 2}\left(\mathbb{R} ; L^{2}\left(\mathbb{S}^{2}\right)\right) \rightarrow$ $H_{\sigma}^{-5 / 2}\left(\mathbb{R} ; L^{2}\left(\mathbb{S}^{2}\right)\right)$ of $\tilde{F}_{\sigma}$ in the $L_{\sigma}^{2}$-duality product defined in (3.4). To obtain the explicit expression of $\tilde{F}_{\sigma}^{*}$ we first formally compute $F_{\sigma}^{*}$. To this end, for smooth compactly supported $g, h$ we have

$$
\begin{aligned}
\left\langle F_{\sigma} g, h\right\rangle & =\int_{\mathbb{S}^{2}} \int_{\mathbb{R}} e^{-2 \sigma t}\left(F_{\sigma} g\right)(\xi, t) \cdot h(\xi, t) \mathrm{d} t \mathrm{~d} \xi \\
& =\int_{\mathbb{S}^{2}} \int_{\mathbb{R}} e^{-2 \sigma t}\left(\int_{\mathbb{S}^{2}} \int_{\mathbb{R}} u_{\sigma}^{\infty}\left(\xi, t-t_{0} ; \theta\right) g\left(\theta, t_{0}\right) d \theta d t_{0}\right) h(\xi, t) \mathrm{d} \xi \mathrm{d} t \\
& =\int_{\mathbb{R}} \int_{\mathbb{S}^{2}} g\left(\theta, t_{0}\right) e^{-2 \sigma t_{0}}\left(\int_{\mathbb{S}^{2}} \int_{\mathbb{R}} e^{-2 \sigma\left(t-t_{0}\right)} u_{\sigma}^{\infty}\left(\xi, t-t_{0} ; \theta\right) h(\xi, t) \mathrm{d} \xi \mathrm{d} t\right) \mathrm{d} \theta \mathrm{d} t_{0}
\end{aligned}
$$

If we let

$$
u_{\sigma}^{* \infty}(\xi, t ; \theta):=e^{-2 \sigma t} u_{\sigma}^{\infty}(\theta, t ; \xi)
$$

then $F_{\sigma}^{*}$ takes the form of the following time-convolution integral operator

$$
\left(F_{\sigma}^{*} h\right)(\xi, t):=\int_{\mathbb{R}} \int_{\mathbb{S}^{2}} u_{\sigma}^{* \infty}\left(\xi, t_{0}-t ; \theta\right) h\left(t_{0}, \theta\right) \mathrm{d} t_{0} \mathrm{~d} \theta .
$$

Note that $u_{\sigma}^{* \infty}(\xi, t ; \theta)$ is not a far field pattern of any physical solutions to the wave equation, hence the dual $F_{\sigma}^{*}$ is not a far field operator. Now using a denseness argument and definition $\tilde{F}_{\sigma}^{*}=\left[\left(\partial_{t}-2 \sigma\right) F_{\sigma}\right]^{*}$, we obtain that $\tilde{F}_{\sigma}^{*}: H_{\sigma}^{5 / 2}\left(\mathbb{R} ; L^{2}\left(\mathbb{S}^{2}\right)\right) \rightarrow$ $H_{\sigma}^{-5 / 2}\left(\mathbb{R} ; L^{2}\left(\mathbb{S}^{2}\right)\right)$ is given by

$$
\left(\tilde{F}_{\sigma}^{*} h\right)(\xi, t):=\int_{\mathbb{R}} \int_{\mathbb{S}^{2}} \partial_{t} u_{\sigma}^{* \infty}\left(\xi, t_{0}-t ; \theta\right) h\left(t_{0}, \theta\right) \mathrm{d} t_{0} \mathrm{~d} \theta
$$


Central to the justification of the factorization method is the following coercivity property that forced us to introduce the modified far field operator at the first place.

Lemma 4.2. Let $\sigma>0$ and $T \in \mathbb{R}$ or $T=-\infty$. Define $\mathcal{T}:=\left(\partial_{t}\left(S^{-1}\right)-2 \sigma S^{-1}\right):$ $H_{\sigma}^{3 / 2}\left(\mathbb{R}_{>T} ; H^{1 / 2}(\partial D)\right) \rightarrow H_{\sigma}^{-3 / 2}\left(\mathbb{R}_{>T} ; H^{-1 / 2}(\partial D)\right)$. Then $\mathcal{T}$ satisfies the following coercivity property

$$
\langle\mathcal{T} \psi, \psi\rangle \geq C(\sigma)\|\psi\|_{L_{\sigma}^{2}\left(\mathbb{R} ; H^{1 / 2}(\partial D)\right)}^{2} \quad \text { for all } \psi \in H_{\sigma}^{3 / 2}\left(\mathbb{R}_{>T} ; H^{1 / 2}(\partial D)\right)
$$

where $\langle\cdot, \cdot\rangle$ denotes the $L_{\sigma}^{2}$-duality product defined in (3.4) with $X=H^{1 / 2}(\partial D)$ and $C(\sigma)>0$ such that $C(\sigma) \rightarrow 0$ as $\sigma \rightarrow 0$.

Proof. The result is a direct consequence of [4, Propositions 2 and 4$]$ which state that

$$
-\int_{\mathbb{R}} \exp (-2 \sigma t) \int_{\partial D} S^{-1}(\psi) \partial_{t} \psi \mathrm{d} x \mathrm{~d} t \geq C(\sigma)\|\psi\|_{L_{\sigma}^{2}\left(\mathbb{R} ; H^{1 / 2}(\partial D)\right)}^{2}
$$

for all $\psi \in C_{0}^{\infty}\left(\mathbb{R} ; H^{1 / 2}(\partial D)\right)$. Integrating by part in time then using a density argument yields

$$
\int_{\mathbb{R}} \exp (-2 \sigma t) \int_{\partial D} \partial_{t}\left(S^{-1} \psi\right)-2 \sigma\left(S^{-1} \psi\right) \psi \mathrm{d} x \mathrm{~d} t \geq C(\sigma)\|\psi\|_{L_{\sigma}^{2}\left(\mathbb{R} ; H^{1 / 2}(\partial D)\right)}^{2}
$$

for all $\psi \in H_{\sigma}^{3 / 2}\left(\mathbb{R} ; H^{1 / 2}(\partial D)\right)$.

A corollary of this lemma and Theorem 4.1 is that

$$
\widetilde{F}_{\sigma}=\mathcal{H}_{\sigma}^{*} \mathcal{T H} \mathcal{H}_{\sigma}
$$

and satisfies the following coercivity property

$$
\left\langle\widetilde{F}_{\sigma} g, g\right\rangle \geq C(\sigma)\left\|\mathcal{H}_{\sigma} g\right\|_{L_{\sigma}^{2}\left(\mathbb{R} ; H^{1 / 2}(\partial D)\right)}^{2} \quad \text { for all } g \in H_{\sigma}^{5 / 2}\left(\mathbb{R} ; L^{2}\left(\mathbb{S}^{2}\right)\right)
$$

where $\langle\cdot, \cdot\rangle$ denotes here the $L_{\sigma}^{2}$-duality product defined in $(3.4)$ with $X=L^{2}\left(\mathbb{S}^{2}\right)$.

We now need to deal with the causality property of the fields. To this end, let $\tau>0$ be a fixed parameter and introduce the truncated far field operator

$$
\tilde{F}_{\sigma}^{\tau}:\left.g \mapsto \tilde{F}_{\sigma} g\right|_{t \geq \tau}
$$

Then $\tilde{F}_{\sigma}^{\tau}: H_{\sigma}^{5 / 2}\left(\mathbb{R}_{>\tau} ; L^{2}\left(\mathbb{S}^{2}\right)\right) \rightarrow \tilde{H}_{\sigma}^{-5 / 2}\left(\mathbb{R}_{>\tau} ; L^{2}\left(\mathbb{S}^{2}\right)\right)$ and

$$
\left\langle\tilde{F}_{\sigma}^{\tau} g, g\right\rangle \geq C(\sigma)\left\|\mathcal{H}_{\sigma} g\right\|_{L_{\sigma}^{2}\left(\mathbb{R} ; H^{1 / 2}(\partial D)\right)}^{2} \quad \text { for all } g \in H_{\sigma}^{5 / 2}\left(\mathbb{R}_{>\tau} ; L^{2}\left(\mathbb{S}^{2}\right)\right) \text {. }
$$

Now let $\left(\tilde{F}_{\sigma}^{\tau}\right)^{*}: H_{\sigma}^{5 / 2}\left(\mathbb{R}_{>\tau} ; L^{2}\left(\mathbb{S}^{2}\right)\right) \rightarrow \tilde{H}_{\sigma}^{-5 / 2}\left(\mathbb{R}_{>\tau} ; L^{2}\left(\mathbb{S}^{2}\right)\right)$ be the adjoint of $\tilde{F}_{\sigma}^{\tau}$ with respect to the $L_{\sigma}^{2}$-duality product, which is a composition of $\tilde{F}_{\sigma}^{*}$ given by (4.6) with the zero-extension operator in $H_{\sigma}^{5 / 2}\left(\mathbb{R}_{>\tau} ; L^{2}\left(\mathbb{S}^{2}\right)\right)$. The coercivity property (4.11) shows that the symmetric operator

$$
\tilde{F}_{\sigma}^{\tau}+\left(\tilde{F}_{\sigma}^{\tau}\right)^{*}: H_{\sigma}^{5 / 2}\left(\mathbb{R}_{>\tau} ; L^{2}\left(\mathbb{S}^{2}\right)\right) \rightarrow \tilde{H}_{\sigma}^{-5 / 2}\left(\mathbb{R}_{>\tau} ; L^{2}\left(\mathbb{S}^{2}\right)\right)
$$

is positive. Furthermore, applying Lemma 1.3 with $X=H_{\sigma}^{5 / 2}\left(\mathbb{R}_{>\tau} ; L^{2}\left(\mathbb{S}^{2}\right)\right)$ and $H=L_{\sigma}^{2}\left(\mathbb{R}_{>\tau} ; L^{2}\left(\mathbb{S}^{2}\right)\right)$ we deduce that

$$
\operatorname{Re} \tilde{F}_{\sigma}^{\tau}=\tilde{F}_{\sigma}^{\tau}+\left(\tilde{F}_{\sigma}^{\tau}\right)^{*}=\left(Q_{F}^{\tau}\right)^{*}\left(Q_{F}^{\tau}\right)
$$


for some operator $Q_{F}^{\tau}: H_{\sigma}^{5 / 2}\left(\mathbb{R}_{>\tau} ; L^{2}\left(\mathbb{S}^{2}\right)\right) \rightarrow L_{\sigma}^{2}\left(\mathbb{R}_{>\tau} ; L^{2}\left(\mathbb{S}^{2}\right)\right)$.

Next let us denote by $\mathcal{H}_{\sigma}^{\tau}$ the restriction of $\mathcal{H}_{\sigma}$ to $H_{\sigma}^{5 / 2}\left(\mathbb{R}_{>\tau} ; L^{2}\left(\mathbb{S}^{2}\right)\right)$. Following the proof of Lemma 3.2 we can view the restricted Herglotz operator as a bounded operator between the following spaces

$$
\mathcal{H}_{\sigma}^{\tau}: H_{\sigma}^{5 / 2}\left(\mathbb{R}_{>\tau} ; L^{2}\left(\mathbb{S}^{2}\right)\right) \rightarrow H_{\sigma}^{3 / 2}\left(\mathbb{R}_{>\tau-d} ; H^{1 / 2}(\partial D)\right) .
$$

We then view the operator $\mathcal{T}$ as

$$
\mathcal{T}: H_{\sigma}^{3 / 2}\left(\mathbb{R}_{>\tau-d} ; H^{1 / 2}(\partial D)\right) \rightarrow \tilde{H}_{\sigma}^{-3 / 2}\left(\mathbb{R}_{>\tau-d} ; H^{-1 / 2}(\partial D)\right) .
$$

We also have

$$
\tilde{F}_{\sigma}^{\tau}=\left(\mathcal{H}_{\sigma}^{\tau}\right)^{*} \mathcal{T} \mathcal{H}_{\sigma}^{\tau}
$$

where

$$
\left(\mathcal{H}_{\sigma}^{\tau}\right)^{*} \psi:=\left.4 \pi R \psi\right|_{t \geq \tau}
$$

Indeed from Lemma 4.2

$$
\langle\mathcal{T} \psi, \psi\rangle \geq C(\sigma)\|\psi\|_{L_{\sigma}^{2}\left(\mathbb{R} ; H^{1 / 2}(\partial D)\right)}^{2} \quad \text { for all } \psi \in H_{\sigma}^{3 / 2}\left(\mathbb{R}_{>\tau-d} ; H^{1 / 2}(\partial D)\right) .
$$

Applying Lemma 1.3 with $X=H_{\sigma}^{3 / 2}\left(\mathbb{R}_{>\tau-d} ; L^{2}\left(\mathbb{S}^{2}\right)\right)$ and $H=L_{\sigma}^{2}\left(\mathbb{R}_{>\tau-d} ; L^{2}(\partial D)\right)$ we deduce that

$$
\mathcal{T}+\mathcal{T}^{*}=Q_{\mathcal{T}}^{*} Q_{\mathcal{T}}
$$

for some operator $Q_{\mathcal{T}}: H_{\sigma}^{3 / 2}\left(\mathbb{R}_{>\tau-d} ; H^{1 / 2}(\partial D)\right) \rightarrow L_{\sigma}^{2}\left(\mathbb{R}_{>\tau-d} ; L^{2}(\partial D)\right)$. Here again, the operator $\mathcal{T}^{*}$ is the dual of $\mathcal{T}$ with respect to $L_{\sigma}^{2}\left(\mathbb{R} ; L^{2}(\partial D)\right)$ duality product. We then obtain from (4.9) and Lemma 1.2 that

$$
\text { the ranges of }\left(Q_{F}^{\tau}\right)^{*} \text { and }\left(Q_{\mathcal{T}} \mathcal{H}_{\sigma}^{\tau}\right)^{*} \text { coincide. }
$$

4.1. A Range Test for $D$. We now prove the following important result that relates the domain $D$ to the range of the operator $\mathcal{H}_{\sigma}^{*}$. This characterization relies on special test functions. Let $\chi: \mathbb{R} \rightarrow \mathbb{R}$ be a smooth non trivial function with compact support in time and choose parameters $z \in \mathbb{R}^{3}$ in space. We define a family of test functions $\varphi_{z}^{\infty}$ by

$$
\varphi_{z}^{\infty}(\xi, t):=\frac{1}{4 \pi} \chi(t+\xi \cdot z) \quad \text { for } \xi \in \mathbb{S}^{2} \text { and } t \in \mathbb{R} .
$$

These test functions are nothing but far fields associated with point sources

$$
\varphi_{z}(x, t):=\frac{\chi(t-|x-z|)}{4 \pi|x-z|} \quad \text { for } x \in \mathbb{R}^{3} \backslash\{z\} \text { and } t \in \mathbb{R} .
$$

For $\eta \in \mathbb{R}$ we define

$$
\varphi_{\eta, z}^{\infty}(\xi, t)=\varphi_{z}^{\infty}(\xi, t-\eta) \quad \text { for } \xi \in \mathbb{S}^{2} \text { and } t \in \mathbb{R}
$$

which is the far field associated with

$$
\varphi_{\eta, z}(x, t):=\frac{\chi(t-\eta-|x-z|)}{4 \pi|x-z|} \quad \text { for } x \in \mathbb{R}^{3} \backslash\{z\} \text { and } t \in \mathbb{R} .
$$

We can prove the following result.

Lemma 4.3. Let $\sigma>0$ be a given. The following holds: 
- If $z \in D$ and $\eta$ is such that the support in time of $\varphi_{\eta, z}^{\infty}$ is included in $\mathbb{R}_{>0}$, then there exists a $\psi \in L_{\sigma}^{2}\left(\mathbb{R}_{>0} ; H^{-1 / 2}(\partial D)\right)$ such that $\mathcal{H}_{\sigma}^{*} \psi=\varphi_{\eta, z}^{\infty}$.

- If $z \notin D$ and $\eta \in \mathbb{R}$, then $\mathcal{H}_{\sigma}^{*} \psi \neq \varphi_{\eta, z}^{\infty}$ for all $\psi \in H_{\sigma}^{-3 / 2}\left(\mathbb{R} ; H^{-1 / 2}(\partial D)\right)$.

Proof. First we consider the case when $z \in D$. Fix $\eta$ such that the support in time of $\varphi_{\eta, z}^{\infty}$ is included in $\mathbb{R}_{>0}$ and set $\psi:=S^{-1}\left(\left.\varphi_{\eta, z}\right|_{\partial D \times \mathbb{R}}\right)$. By construction, it holds that $\varphi_{\eta, z}=\mathrm{SL}(\psi)$. Hence, $\varphi_{\eta, z}^{\infty}=R(\psi)=(4 \pi)^{-1} \mathcal{H}_{\sigma}^{*} \psi$. From the assumption the support in time of $\left.\varphi_{\eta, z}\right|_{\partial D \times \mathbb{R}}$ is necessarily included in $\mathbb{R}_{>0}$. Therefore $\left.\varphi_{\eta, z}\right|_{\partial D \times \mathbb{R}} \in$ $H_{\sigma}^{m}\left(\mathbb{R}_{>0} ; H^{1 / 2}(\partial D)\right)$ for any positive $m$. We obtain in particular from Theorem 3.1 that $\psi \in L_{\sigma}^{2}\left(\mathbb{R}_{>0} ; H^{-1 / 2}(\partial D)\right)$ which proves the first part of the lemma.

Now we consider the case when $z \notin D$. Let $\eta \in \mathbb{R}$ and assume that $4 \pi \varphi_{\eta, z}^{\infty}=\mathcal{H}_{\sigma}^{*} \psi$ for some $\psi \in H_{\sigma}^{-3 / 2}\left(\mathbb{R} ; H^{-1 / 2}(\partial D)\right)$. Applying the Laplace transform to this equality implies

$$
4 \pi \int_{\partial D} \exp (-\mathrm{i} k \xi \cdot x) \mathcal{L}[\psi](x, k) \mathrm{d} s_{x}=e^{i k \tau} \mathcal{L}[\chi](k) \exp (-\mathrm{i} k \xi \cdot z), \quad \xi \in \mathbb{S}^{2}
$$

for $k:=\omega+\mathrm{i} \sigma, \omega \in \mathbb{R}$. Set $\hat{\psi}(x, k):=\mathcal{L}[\psi](x, k)$ and denote the single-layer potential at frequency $k=\omega+\mathrm{i} \sigma$, by

$$
\hat{\mathrm{SL}}(k) \hat{\psi}=\int_{\partial D} \frac{\exp (\mathrm{i} k|\cdot-y|)}{4 \pi|\cdot-y|} \hat{\psi}(y) \mathrm{d} y .
$$

for $\hat{\psi} \in H^{-1 / 2}(\partial D)$. If equality (4.19) holds then, using the Rellich Lemma for complex wave numbers [40] and a unique continuation principle, we obtain that

$$
\hat{\mathrm{SL}}(k) \hat{\psi}(\cdot, k)=e^{i k \tau} \mathcal{L}[\chi](k) \frac{\exp (\mathrm{i} k|\cdot-z|)}{4 \pi|\cdot-z|} \quad \text { in } \mathbb{R}^{3} \backslash D .
$$

Indeed the latter cannot hold if $\mathcal{L}[\chi](k) \neq 0$ since the right hand side does not belong to $H_{\text {loc }}^{1}\left(\mathbb{R}^{3} \backslash D\right)$ while the left hand side does. Consequently (4.19) cannot hold for any $k$ such that $\mathcal{L}[\chi](k) \neq 0$. Therefore for every $\eta \in \mathbb{R}, \varphi_{\eta, z}^{\infty} \neq \mathcal{H}_{\sigma}^{*} \psi$ for all $\psi \in H_{\sigma}^{-3 / 2}\left(\mathbb{R} ; H^{-1 / 2}(\partial D)\right)$. This proves the second part of the lemma.

Remark 4.4. Proving the second part of Lemma 4.3 for $\left(\mathcal{H}_{\sigma}^{\tau}\right)^{*}$ for a fixed $\tau$, in other words showing that, for $\eta$ such that the support in time of $\varphi_{\eta, z}^{\infty}$ is included in $\mathbb{R}_{>0}$, there exists a $\psi \in L_{\sigma}^{2}\left(\mathbb{R}_{>0} ; H^{-1 / 2}(\partial D)\right)$ such that $\mathcal{H}_{\sigma}^{*} \psi=\varphi_{\eta, z}^{\infty}$ if and only if $z \in D$, is an open problem. Such result seems to be related to the geometry of $D$, and if available will simplify the range test stated in the main theorem below. More specifically, in this case the result of Theorem 4.5 would hold for a fixed $\tau$ and no finite supremum condition is needed.

We are now in position to state and prove the main result of this section. To this end we recall that $\left(Q_{F}^{\tau}\right)^{*}: L_{\sigma}^{2}\left(\mathbb{R}_{>\tau} ; L^{2}\left(\mathbb{S}^{2}\right)\right) \rightarrow \tilde{H}_{\sigma}^{-5 / 2}\left(\mathbb{R}_{>\tau} ; L^{2}\left(\mathbb{S}^{2}\right)\right)$ is the dual operator of $Q_{F}^{\tau}: H_{\sigma}^{5 / 2}\left(\mathbb{R}_{>\tau} ; L^{2}\left(\mathbb{S}^{2}\right)\right) \rightarrow L_{\sigma}^{2}\left(\mathbb{R}_{>\tau} ; L^{2}\left(\mathbb{S}^{2}\right)\right)$ given by (4.12) with respect to $L_{\sigma}^{2}\left(\mathbb{R} ; L^{2}(\partial D)\right)$ duality product. Then we can prove the following result.

TheOREM 4.5. Let $\sigma>0$ be a given parameter, $z \in \mathbb{R}^{3}$ and assume that $\eta_{z} \in \mathbb{R}$ (fixed but $z$-dependent) is such that the support in time of $\varphi_{\eta_{z}, z}^{\infty}$ is included in $\mathbb{R}_{>0}$. Then for $\tau \in \mathbb{R}, \varphi_{\eta_{z}, z}^{\infty}=\left(Q_{F}^{\tau}\right)^{*} \varrho_{\tau}$ with $\varrho_{\tau} \in L_{\sigma}^{2}\left(\mathbb{R}_{>\tau} ; L^{2}\left(\mathbb{S}^{2}\right)\right)$ such that $\sup _{\tau \leq 0}\left\|\varrho_{\tau}\right\|_{L_{\sigma}^{2}\left(\mathbb{R}_{>\tau} ; L^{2}\left(\mathbb{S}^{2}\right)\right)}<+\infty$ if and only if $z \in D$. 
Proof. First we remark that we have already seen that the range of $\left(Q_{F}^{\tau}\right)^{*}$ coincides with the range of $\left(\mathcal{H}_{\sigma}^{\tau}\right)^{*} Q_{\mathcal{T}}^{*}$, where we recall that $\left(Q_{\mathcal{T}}\right)^{*}: L_{\sigma}^{2}\left(\mathbb{R}_{>\tau-d} ; L^{2}(\partial D)\right) \rightarrow$ $\tilde{H}_{\sigma}^{-3 / 2}\left(\mathbb{R}_{>\tau-d} ; H^{-1 / 2}(\partial D)\right)$ is given by $(4.13)$, and $\left(\mathcal{H}_{\sigma}^{\tau}\right)^{*}: \tilde{H}_{\sigma}^{-3 / 2}\left(\mathbb{R}_{>\tau-d} ; H^{-1 / 2}(\partial D)\right) \rightarrow$ $\tilde{H}_{\sigma}^{-5 / 2}\left(\mathbb{R}_{>\tau} ; L^{2}\left(\mathbb{S}^{2}\right)\right)$.

We now consider the case when $z \in D$. From the first part of Lemma 4.3, there exists $\psi \in L_{\sigma}^{2}\left(\mathbb{R}_{>0} ; H^{-1 / 2}(\partial D)\right)$ such that $\mathcal{H}_{\sigma}^{*} \psi=\varphi_{\eta_{z}, z}^{\infty}$. We observe that the extension by 0 of $\psi$ in the time half line $\mathbb{R}_{<0}$ provides a function $\psi \in L_{\sigma}^{2}\left(\mathbb{R}_{>\tau} ; H^{-1 / 2}(\partial D)\right)$ such that $\left(\mathcal{H}_{\sigma}^{\tau}\right)^{*} \psi=\varphi_{\eta_{z}, z}^{\infty}$. Let $g \in H_{\sigma}^{5 / 2}\left(\mathbb{R}_{>\tau} ; L^{2}\left(\mathbb{S}^{2}\right)\right)$ such that $\left\langle\varphi_{\eta_{z}, z}^{\infty}, g\right\rangle=1$ in the $L_{\sigma}^{2}$ duality. Then

$$
\begin{aligned}
\left\|\mathcal{H}_{\sigma} g\right\|_{L_{\sigma}^{2}\left(\mathbb{R} ; H^{1 / 2}(\partial D)\right)}^{2} & \geq \frac{\left\langle\psi, \mathcal{H}_{\sigma} g\right\rangle}{\|\psi\|_{L_{\sigma}^{2}\left(\mathbb{R}_{>0} ; H^{-1 / 2}(\partial D)\right)}}=\frac{\left\langle\varphi_{\eta_{z}, z}^{\infty}, g\right\rangle}{\|\psi\|_{L_{\sigma}^{2}\left(\mathbb{R}_{>0} ; H^{-1 / 2}(\partial D)\right)}} \\
& =\frac{1}{\|\psi\|_{L_{\sigma}^{2}\left(\mathbb{R}_{>0} ; H^{-1 / 2}(\partial D)\right)}} .
\end{aligned}
$$

Now the inequality (4.11) implies that

$$
\left\langle\operatorname{Re} \tilde{F}_{\sigma}^{\tau} g, g\right\rangle \geq C(\sigma)\left\|\mathcal{H}_{\sigma} g\right\|_{L_{\sigma}^{2}\left(\mathbb{R} ; H^{1 / 2}(\partial D)\right)}^{2} \quad \text { for all } g \in H_{\sigma}^{5 / 2}\left(\mathbb{R}_{>\tau} ; L^{2}\left(\mathbb{S}^{2}\right)\right),
$$

hence since $C(\sigma)$ is independent of $\tau \in \mathbb{R}$, we obtain

$$
\inf _{\tau \leq 0} \inf _{g \in X_{\tau}}\left\langle\operatorname{Re} \tilde{F}_{\sigma}^{\tau} g, g\right\rangle \geq C(\sigma) \frac{1}{\|\psi\|_{L_{\sigma}^{2}\left(\mathbb{R}_{>0} ; H^{-1 / 2}(\partial D)\right)}}>0
$$

where

$$
X_{\tau}:=\left\{g \in H_{\sigma}^{5 / 2}\left(\mathbb{R}_{>\tau} ; L^{2}\left(\mathbb{S}^{2}\right)\right) ;\left\langle g, \varphi_{\eta_{z}, z}^{\infty}\right\rangle=1\right\} .
$$

Then using Theorem A.1 with $F:=\operatorname{Re} \tilde{F}_{\sigma}^{\tau}, H:=Q_{F}^{\tau}$ and $T:=I$, we conclude that $\varphi_{\eta_{z}, z}^{\infty}$ is in the range of $\left(Q_{F}^{\tau}\right)^{*}$ for all $\tau \in \mathbb{R}$, i.e. $\varphi_{\eta_{z}, z}^{\infty}=\left(Q_{F}^{\tau}\right)^{*} \varrho_{\tau}$ with $\varrho_{\tau} \in$ $L_{\sigma}^{2}\left(\mathbb{R}_{>\tau} ; L^{2}\left(\mathbb{S}^{2}\right)\right)$. Furthermore, reasoning in the same way as above (see also the proof of the first part of Theorem A.1) we obtain for each $\tau \in \mathbb{R}$ and $g \in X_{\tau}$

$$
\left\langle\operatorname{Re} \tilde{F}_{\sigma}^{\tau} g, g\right\rangle=\left\|Q_{F}^{\tau} g\right\|^{2}
$$

On the other hand

$$
\left\|Q_{F}^{\tau} g\right\| \geq \frac{1}{\left\|\varrho_{\tau}\right\|_{L_{\sigma}^{2}\left(\mathbb{R}_{>\tau} ; L^{2}\left(\mathbb{S}^{2}\right)\right)}}\left\langle\varrho_{\tau}, Q_{F}^{\tau} g\right\rangle=\frac{1}{\left\|\varrho_{\tau}\right\|_{L_{\sigma}^{2}\left(\mathbb{R}_{>\tau} ; L^{2}\left(\mathbb{S}^{2}\right)\right)}^{2}},
$$

with the equality holding for $g$ and $\varphi_{\eta_{z}, z}^{\infty}$ linearly dependent. Thus

$$
\inf _{g \in X_{\tau}}\left\langle\operatorname{Re} \tilde{F}_{\sigma}^{\tau} g, g\right\rangle=\frac{1}{\left\|\varrho_{\tau}\right\|_{L_{\sigma}^{2}\left(\mathbb{R}_{>\tau} ; L^{2}\left(\mathbb{S}^{2}\right)\right)}^{2}} .
$$

But since from (4.20) the infimum over $\tau \leq 0$ of the left hand side is positive, then

$$
\sup _{\tau \leq 0}\left\|\varrho_{\tau}\right\|_{L_{\sigma}^{2}\left(\mathbb{R}_{>\tau} ; L^{2}\left(\mathbb{S}^{2}\right)\right)}<+\infty .
$$

Next consider the case when $z \notin D$. Assume that for each $\tau \in \mathbb{R}, \varphi_{\eta_{z}, z}^{\infty}=\left(\mathcal{H}_{\sigma}^{\tau}\right)^{*} \psi_{\tau}$ 
for some $\psi_{\tau} \in \tilde{H}_{\sigma}^{-3 / 2}\left(\mathbb{R}_{>\tau-d} ; H^{-1 / 2}(\partial D)\right)$ and $\sup _{\tau \leq 0}\left\|\psi_{\tau}\right\|_{\tilde{H}_{\sigma}^{-3 / 2}\left(\mathbb{R}_{>\tau-d} ; H^{-1 / 2}(\partial D)\right)}<$ $+\infty$. The latter implies the existence of $\psi \in H_{\sigma}^{-3 / 2}\left(\mathbb{R} ; H^{-1 / 2}(\partial D)\right)$ such that $\left(\mathcal{H}_{\sigma}\right)^{*} \psi=$ $\varphi_{\eta_{z}, z}^{\infty}$, this is not possible because it contradicts the second part of Lemma 4.3. Let us show that under the above assumption such a $\psi$ exist: We denote by $\tilde{\psi}_{\tau} \in$ $H_{\sigma}^{-3 / 2}\left(\mathbb{R} ; H^{-1 / 2}(\partial D)\right)$ be an extension of $\psi_{\tau}$ satisfying $\left\|\tilde{\psi}_{\tau}\right\|_{H_{\sigma}^{-3 / 2}\left(\mathbb{R} ; H^{-1 / 2}(\partial D)\right)} \leq$ $\left\|\psi_{\tau}\right\|_{H_{\sigma}^{-3 / 2}\left(\mathbb{R}_{>\tau-d} ; H^{-1 / 2}(\partial D)\right)}+1 /|\tau|$. Then $\sup _{\tau}\left\|\tilde{\psi}_{\tau}\right\|_{H_{\sigma}^{-3 / 2}\left(\mathbb{R} ; H^{-1 / 2}(\partial D)\right)}<+\infty$ and therefore (the space is reflexive) there exists $\psi \in H_{\sigma}^{-3 / 2}\left(\mathbb{R} ; H^{-1 / 2}(\partial D)\right.$ ) such that (up to a subsequence) $\tilde{\psi}_{\tau}$ weakly converges to $\psi$ in $H_{\sigma}^{-3 / 2}\left(\mathbb{R} ; H^{-1 / 2}(\partial D)\right)$. Now, for a compactly supported function smooth $\varphi$, we have that

$$
\left\langle\varphi_{\eta_{z}, z}^{\infty}, \varphi\right\rangle=\left\langle\left(\mathcal{H}_{\sigma}^{\tau}\right)^{*} \psi_{\tau}, \varphi\right\rangle=\left\langle\psi_{\tau},\left(\mathcal{H}_{\sigma}^{\tau}\right) \varphi\right\rangle=\left\langle\tilde{\psi}_{\tau}, \mathcal{H}_{\sigma} \varphi\right\rangle
$$

where we assume $\varphi(t, x)=0$ for $t<\tau_{0}-d$ for small enough $\tau_{0}<0$ and $\langle\cdot, \cdot\rangle$ is the $L_{\sigma}^{2}$-duality. Now letting $\tau \rightarrow-\infty$ in the above we have that

$$
\left\langle\varphi_{\eta_{z}, z}^{\infty}, \varphi\right\rangle=\left\langle\psi_{\tau}, \mathcal{H}_{\sigma} \varphi\right\rangle=\left\langle\mathcal{H}_{\sigma}^{*} \psi, \varphi\right\rangle
$$

and by a denseness argument this holds for all $\varphi \in H_{\sigma}^{3 / 2}\left(\mathbb{R} ; H^{1 / 2}(\partial D)\right)$ implying that $\left(\mathcal{H}_{\sigma}\right)^{*} \psi=\varphi_{\eta_{z}, z}^{\infty}$. Therefore the following two possibilities can happen: 1$)$ either there is a $\tau_{0} \in \mathbb{R}$ for which $\varphi_{\eta_{z}, z}^{\infty}$ is not in the range of $\left(\mathcal{H}_{\sigma}^{\tau_{0}}\right)^{*}$, or 2) for all $\tau \in \mathbb{R}, \varphi_{\eta_{z}, z}^{\infty}$ is in the range of $\left(\mathcal{H}_{\sigma}^{\tau}\right)^{*}$ but $\sup _{\tau \leq 0}\left\|\psi_{\tau}\right\|_{\tilde{H}_{\sigma}^{-3 / 2}\left(\mathbb{R}_{>\tau-d} ; H^{-1 / 2}(\partial D)\right)}=+\infty$. In the case 1) there is nothing to prove since it means that there exists $\tau_{0} \in \mathbb{R}$ such that $\varphi_{\eta_{z} z}^{\infty}$ is not in the range of $\left(Q_{F}^{\tau_{0}}\right)^{*}$. In the case 2$)$, for each fixed $\tau \in \mathbb{R}$ we have that $\varphi_{\eta_{z}, z}^{\infty}$ is in the range of $\left(Q_{F}^{\tau}\right)^{*}$, i.e. $\varphi_{\eta_{z}, z}^{\infty}=\left(Q_{F}^{\tau}\right)^{*} \varrho_{\tau}$ with $\varrho_{\tau} \in L_{\sigma}^{2}\left(\mathbb{R}_{>\tau} ; L^{2}\left(\mathbb{S}^{2}\right)\right)$. Exactly in the same way as in the proof of the first part we have that for every $\tau \in \mathbb{R}$

$$
\inf _{g \in X_{\tau}}\left\langle\operatorname{Re} \tilde{F}_{\sigma}^{\tau} g, g\right\rangle=\frac{1}{\left\|\varrho_{\tau}\right\|_{L_{\sigma}^{2}\left(\mathbb{R}_{>\tau} ; L^{2}\left(\mathbb{S}^{2}\right)\right)}^{2}}
$$

where $X_{\tau}$ is defined by (4.21).

On the other hand, again applying the inf-criterion, i.e. Theorem A.1, to the factorization $\tilde{F}_{\sigma}^{\tau}=\left(\mathcal{H}_{\sigma}^{\tau}\right)^{*} \mathcal{T} \mathcal{H}_{\sigma}^{\tau}$, using the coercivity property of the operator $\mathcal{T}$ with coercivity constant independent of $\tau$, and the fact that $\sup _{\tau \leq 0}\left\|\psi_{\tau}\right\|_{\tilde{H}_{\sigma}^{-3 / 2}\left(\mathbb{R}_{>\tau-d} ; H^{-1 / 2}(\partial D)\right)}=$ $+\infty$ we have that

$$
\inf _{\tau \leq 0} \inf _{g \in X_{\tau}}\left\langle\operatorname{Re} \tilde{F}_{\sigma}^{\tau} g, g\right\rangle=0
$$

which together with $(4.22)$ implies that $\sup _{\tau \leq 0}\left\|\varrho_{\tau}\right\|_{L_{\sigma}^{2}\left(\mathbb{R}_{>\tau} ; L^{2}\left(\mathbb{S}^{2}\right)\right)}=+\infty$. This end the proof of the theorem.

We conclude with the following remarks addressing the challenging task of letting $\sigma \rightarrow 0$ as well as possible numerical implementation of our range test.

4.2. Conclusions. The rigorous range test for determining $D$ is proven for the operator $F_{\sigma}$ which is not available from the measured data. As already explained, this is because $F_{\sigma}$ involves the far field of the scattered fields due to non-physical incident waves $u_{\sigma}^{i}(x, t ; \theta):=\delta(t-\theta \cdot x) e^{2 \sigma(\theta \cdot x)}$ which aren't solutions to the wave equations (their Laplace transform solves the Helmholtz equation $\Delta v+(\omega-\mathrm{i} \sigma)^{2} v=0$ ), but 
approach the physical wave fronts $u_{\sigma}^{i}(x, t ; \theta):=\delta(t-\theta \cdot x)$ as $\sigma \rightarrow 0$. Therefore in the limiting case of $\sigma \rightarrow 0$, as mentioned earlier one can easily see that, at least formally, $F_{\sigma}$ approaches the physical far field operator $F$. However, it is impossible in our analysis to let $\sigma \rightarrow 0$ because the fundamental coercivity property in Lemma 4.2 does not hold for $\sigma=0$ due to the fact that the coercivity constant $C(\sigma) \rightarrow 0$. Nevertheless, when implementing the range test of Theorem 4.5, it is reasonable to check if $\varphi_{\eta_{z}, z}^{\infty}$ is in the range of the square root of the operator $\partial_{t} F^{\tau}+\left(\partial_{t} F^{\tau}\right)^{*}$ where $F^{\tau}$ is the restriction of $F$ to causal functions which are zero in $(-\infty, \tau)$ for fixed $\tau<0$ small enough.

Concluding, despite the significant step forward that our analysis makes toward a mathematically rigorous characterization of the support $D$ in terms of time domain data, this question is still not completely resolved. It is highly desirable to investigate convergence of the range test as $\sigma \rightarrow 0$. The generalized linear sampling method developed in the frequency domain in $[1,2]$ (see also [12]), could provide a mathematical framework for such convergence, but unfortunately at this time we are not able to resolve it. An acceptable approach, especially from computational point of view, could be to find a computable way to approximate the perturbed far field operator $F_{\sigma}$ from the physical far field operator $F$, in a similar way as it is being done for the justification of the factorization method in the time domain with near field data [20] or limited aperture data [3].

Acknowledgments. F. C. acknowledges the support of AFOSR Grant FA955017-1-0147, NSF Grants DMS-1602802 and DMS-1813492. Part of this work was done while F.C. visited CMAP at Ecole Polytechnique, and the support of INRIA Saclay and Ecole Polytechnique is kindly acknowledged.

Appendix A. Auxiliary Abstract Results. We state and prove here some abstract results we have used in the paper. We start with a range characterization result known as inf-criterion proven [12], [29]. To this end, let $X$ and $Y$ be two (complex) reflexive Banach spaces with duals $X^{*}$ and $Y^{*}$ respectively and denote by $\langle$,$\rangle a duality product that refers to \left\langle X^{*}, X\right\rangle$ or $\left\langle Y^{*}, Y\right\rangle$ duality. We consider three bounded operators $F: X \rightarrow X^{*}, H: X \rightarrow Y$ and $T: Y \rightarrow Y^{*}$ such that

$$
F=H^{*} T H .
$$

We then have the following range characterization theorem.

TheOREM A.1. Assume that there exists a constant $\alpha>0$ such that

$$
|\langle T \varphi, \varphi\rangle| \geq \alpha\|\varphi\|_{Y}^{2} \quad \forall \varphi \in \mathcal{R}(H) .
$$

Then one has the following characterization of the range of $H^{*}$ :

$$
\left\{\psi^{*} \in \mathcal{R}\left(H^{*}\right) \text { and } \psi^{*} \neq 0\right\} \quad \text { iff } \quad \inf \left\{|\langle F \psi, \psi\rangle|, \psi \in X,\left\langle\psi^{*}, \psi\right\rangle=1\right\}>0
$$

Proof. We first observe that

$$
|\langle F \psi, \psi\rangle|=\left|\left\langle H^{*} T H \psi, \psi\right\rangle\right|=|\langle T H \psi, H \psi\rangle| .
$$

Hence,

$$
\alpha\|H \psi\|_{Y}^{2} \leq|\langle F \psi, \psi\rangle| \leq\|T\|\|H \psi\|_{Y}^{2} \quad \forall \psi \in X .
$$


Let $\psi^{*} \in \mathcal{R}\left(H^{*}\right)$ and $\psi^{*} \neq 0$. Then $\psi^{*}=H^{*}\left(\varphi^{*}\right)$ for some $\varphi^{*} \in Y^{*}$ and $\varphi^{*} \neq 0$. Let $\psi \in X$ such that $\left\langle\psi^{*}, \psi\right\rangle=1$. Then

$$
\begin{aligned}
\|H \psi\|_{Y} & =\frac{1}{\left\|\varphi^{*}\right\|_{Y^{*}}}\|H \psi\|_{Y}\left\|\varphi^{*}\right\|_{Y^{*}} \\
& \geq \frac{1}{\left\|\varphi^{*}\right\|_{Y^{*}}}\left\langle\varphi^{*}, H \psi\right\rangle=\frac{1}{\left\|\varphi^{*}\right\|_{Y^{*}}}>0 .
\end{aligned}
$$

We then deduce, using the first inequality in (A.2), that

$$
\inf \left\{|\langle F \psi, \psi\rangle|, \psi \in X,\left\langle\psi^{*}, \psi\right\rangle=1\right\} \geq \frac{\alpha}{\left\|\varphi^{*}\right\|_{Y^{*}}^{2}}>0 .
$$

Now assume that $\psi^{*} \notin \mathcal{R}\left(H^{*}\right)$ and let us show that

$$
\inf \left\{|\langle F \psi, \psi\rangle|, \psi \in X,\left\langle\psi^{*}, \psi\right\rangle=1\right\}=0 .
$$

From the second inequality in (A.2) it is sufficient to prove the existence of a sequence $\psi_{n} \in X$ such that $\left\langle\psi^{*}, \psi_{n}\right\rangle=1$ and $\left\|H \psi_{n}\right\|_{Y} \rightarrow 0$ as $n \rightarrow \infty$. Since $\psi^{*} \neq 0$ and $X$ is reflexive, there exists $\hat{\psi} \in X$ such that $\left\langle\psi^{*}, \hat{\psi}\right\rangle=1$. Setting $\hat{\psi}_{n}=\hat{\psi}-\psi_{n}$, we see that it is sufficient to show the existence of a sequence $\hat{\psi}_{n} \in X$ such that

$$
\left\langle\psi^{*}, \hat{\psi}_{n}\right\rangle=0 \quad \text { and } \quad H \hat{\psi}_{n} \rightarrow H \hat{\psi} \text { in } Y .
$$

Set $V=\left\{\psi \in X ;\left\langle\psi^{*}, \psi\right\rangle=0\right\}=\left\{\psi^{*}\right\}^{\perp}$ (where the orthogonality is to be understood in the sense of the $X^{*}, X$ duality product). Since $H \hat{\psi} \in \mathcal{R}(H)$, in order to prove (A.3) it is sufficient to prove that $H(V)$ is dense in $\mathcal{R}(H)$ and for the latter it is sufficient to prove (since $Y$ is reflexive) that $H(V)^{\perp}=\mathcal{R}(H)^{\perp}$ (where the orthogonality is to be understood in the sense of the $Y^{*}, Y$ duality product). But this equality follows from

$$
\varphi^{*} \in H(V)^{\perp} \text { if and only if } H^{*} \varphi^{*} \in V^{\perp}=\operatorname{Vect}\left\{\psi^{*}\right\}
$$

hence $H^{*} \varphi^{*}=0$ (since $\left.\psi^{*} \notin \mathcal{R}\left(H^{*}\right)\right)$ meening $\varphi^{*} \in \operatorname{Kern}\left(H^{*}\right)=\mathcal{R}(H)^{\perp}$.

As a corollary we also have the following well-known result on range identities (see also [28]).

Lemma 1.2. Let $X, H_{1}$, and $H_{2}$ be separable Hilbert spaces. Assume that $Q_{1}$ : $X \rightarrow H_{1}$ and $Q_{2}: X \rightarrow H_{2}$ are bounded operators with adjoints $Q_{1,2}^{*}: H_{1,2} \rightarrow X^{*}$, defined by

$$
\left\langle Q_{1,2} u, v_{1,2}\right\rangle_{H_{1,2}}=\left\langle u, Q_{1,2}^{*} v_{1,2}\right\rangle_{X \times X^{*}} \quad \text { for all } u \in X \text { and } v_{1,2} \in H_{1,2} .
$$

If $Q_{1}^{*} Q_{1}=Q_{2}^{*} Q_{2}$, then the ranges of the adjoints $Q_{1}^{*}$ and $Q_{2}^{*}$ coincide in $X^{*}$. We also use the following abstract result on the square root of symmetric positive operators.

Lemma 1.3. Let $X \subset H \subset X^{*}$ be a Gelfand triple with separable Hilbert spaces $H$ and $X$ and assume that $T$ is a bounded, symmetric and non-negative operator from $X$ into $X^{*}$. Then there exists a bounded operator $Q: X \rightarrow H$ such that $T=Q^{*} Q$.

Proof. Let us introduce an isometric Hilbert space isomorphism $J$ from $H$ onto $X$. (Note that both spaces are separable and hence such an isomorphism exists.) The adjoint $J^{*}$ is then an isometry from $X^{*}$ onto $H$. Consequently, $J^{*} \circ T \circ J$ is a bounded symmetric and non-negative operator on $H$,

$$
\left.\left\langle J^{*}(T(J \varphi)), \varphi\right\rangle_{H}=\langle T(J \varphi)), J \varphi\right\rangle_{H} \geq 0
$$


for all $\varphi \in H$. From Theorem 12.32 in [37] we know that a bounded, symmetric and non-negative operator on a Hilbert space is self-adjoint and possesses a unique bounded and self-adjoint square root $Q_{0}$. Let us define $Q=Q_{0} J^{-1}$, a bounded operator from $X$ into $H$. Then $T=Q^{*} Q$.

\section{REFERENCES}

[1] L. Audibert, Qualitative Methods for Heterogeneous Media, PhD thesis, École Polytechnique, Palaiseau, France, 2015.

[2] L. Audibert and H. Haddar, A generalized formulation of the linear sampling method with exact characterization of targets in terms of far field measurements, Inverse Problems 30 paper 035011 (2014).

[3] L. Audibert and H. Haddar, The generalized linear sampling method for limited aperture measurements, SIAM J. Imaging Sci. 1084517870 (2017).

[4] A. Bamberger and T. Ha Duong, Formulation variationelle espace-temps pour le calcul par potentiel retardé de la diffraction d'une onde acoustique, Math. Meth. Appl. Sci., 8 405-435 (1986).

[5] C. Bardos and M. Fink, Mathematical foundations of the time reversal mirror, Asymptot. Anal., 29 157-182 (2002).

[6] G. Beylkin and R. Burridge, Linearized inverse scattering problems in acoustics and elasticity. Wave Motion, 12 15-52 (1990).

[7] K. Bingham, Y. Kurylev, M. Lassas, and S. Siltanen, Iterative time-reversal control for inverse problems, Inverse Problems and Imaging, 2 63-81 (2008).

[8] N. Bleistein, J. K. Cohen and J. W. Jr. Stockwell, Mathematics of Multidimensional Seismic Imaging, Migration, and Inversion Springer, 2001.

[9] L. Borcea, G. Papanicolaou and C. Tsogka, Adaptive interferometric imaging in clutter and optimal illumination. Inverse Problems, 22 405-1436 (2006).

[10] C. Burkard and R. Potthast. A time-domain probe method for three-dimensional rough surface reconstructions. Inverse Problems and Imaging, 3 259-274 (2009).

[11] F. Cakoni and D. Colton. Qualitative Methods in Inverse Scattering Theory. An Introduction. Springer, Berlin, 2006.

[12] F. Cakoni, D. Colton and H. Haddar, Inverse Scattering Theory and Transmission Eigenvalues, CBMS Series, SIAM Publications, 882016.

[13] F. Cakoni, P. Monk and V. Selgas, Analysis of the linear sampling method for imaging penetrable obstacles in the time domain, (submitted).

[14] F. Cakoni and J. Rezac, Direct imaging of small scatterers using reduced time dependent data, J. Comp. Physics 338, 371-387 (2017).

[15] Q. Chen, H. Haddar, A. Lechleiter and P. Monk, A sampling method for inverse scattering in the time domain. Inverse Problems, 26085001 (2010).

[16] D. L. Colton and R. Kress, Inverse acoustic and electromagnetic scattering theory. Third edition, Springer, 2012.

[17] M. Fink, Time reversal of ultrasonic fields: Basic principles, IEEE Trans. Ultrason., Ferroelectric and Frequency Control, 39 555-566 (1992).

[18] F. G. Friedlander, On the radiation field of pulse solutions of the wave equation, Proc. R. Soc. Lon. A, 269 53-65 (1962).

[19] F. G. Friedlander, On the radiation field of pulse solutions of the wave equation II, Proc. R. Soc. Lon. A, 279 386-394 (1963)

[20] G. Hu, J. Yang, B. Zhang, H. Zhang, Near-field imaging of scattering obstacles with the factorization method, Inverse Problems 30095005 (2014).

[21] Y. Guo, P. Monk and D. Colton, Toward a time domain approach to the linear sampling method, Inverse Problems 29095016 (2013).

[22] Y. Guo, P. Monk and D. Colton, The linear sampling method for sparse small aperture data, Applicable Analysis 95 1599-1615 (2015).

[23] B. Guzina, F. Cakoni, and C. Bellis. On the multi-frequency obstacle reconstruction via the linear sampling method, Inverse Problems, 26125005 (2010).

[24] H. Haddar, A, Lechleiter and S. Marmorat, An improved time domain linear sampling method for Robin and Neumann obstacles, Appl. Anal. 93 369-390 (2014).

[25] M. Ikehata, The enclosure method for inverse obstacle scattering over a finite time interval: IV. Extraction from a single point on the graph of the response operator, J. Inverse Ill-Posed Probl. 25 747-761, (2017). 
[26] M. Ikehata, On finding an obstacle embedded in the rough background medium via the enclosure method in the time domain, Inverse Problems 31085011 (2015).

[27] A. Kirsch, Characterization of the shape of a scattering obstacle using the spectral data of the far field operator, Inverse Problems, 14 1489-1512 (1998).

[28] A. Kirsch, New characterizations of solutions in inverse scattering theory, Applicable Analysis, 76 319-350 (2000).

[29] A. Kirsch and N.I. Grinberg, The Factorization Method for Inverse Problems, Oxford Lecture Series in Mathematics and its Applications 36. Oxford University Press, 2008.

[30] M. Lassas and L. Oksanen, An inverse problem for a wave equation with sources and observations on disjoint sets, Inverse Problems 26085012 (2010).

[31] C. Lubich, On the multistep time discretization of linear initial-boundary value problems and their boundary integral equations, Numer. Math., 267 365-389 (1994).

[32] D. R. Luke and R. Potthast, The point source method for inverse scattering in the time domain, Math. Meth. Appl. Sci., 29 1501-1521 (2006).

[33] L. Oksanen, Inverse obstacle problem for the non-stationary wave equation with an unknown background, Comm. Partial Differential Equations 381492171518 (2013).

[34] L. Oksanen, Solving an inverse obstacle problem for the wave equation by using the boundary control method, Inverse Problems 29035004 (2013).

[35] F. D. Philippe, C. Prada, D. Clorennec, M. Fink, and T. Folégot, Construction of the temporal invariants of the time-reversal operator, JASA Express Letters, 126 EL8-EL13 (2009).

[36] R. Potthast, A survey on sampling and probe methods for inverse problems, Inverse Problems, 22 R1-R47 (2006).

[37] W. Rudin, Functional Analysis, McGraw-Hill, 2. edition, 1991.

[38] F. J. Sayas, Retarded Potentials and Time Domain Boundary Integral Equations. A Road Map. Springer Series in Computational Mathematics, 50. Springer, 2016.

[39] P. Tietäväinen, A Factorization Method for the Inverse Scattering of the Wave Equation, $\mathrm{PhD}$ Thesis, Aalto University, 662011.

[40] C. H. Wilcox, A generalization of theorems of Rellich and Atkinson, Proc. Amer. Math. Soc. 727117276 (1956). 1956. 